\title{
Detection of Malnutrition in Older Infants by Measurement of Mid arm Circumference
}

\author{
Nadkarni $\mathbf{J}^{1}$, Sharma $\mathbf{U}^{2}$, Bagde $\mathbf{R}^{3}$, Dwivedi $\mathbf{R}^{4}$ \\ ${ }^{1}$ Jayashree Nadkarni MD Associate Professor, ${ }^{2}$ Utkarsh Sharma MBBS Intern, ${ }^{3}$ Ravindra Bagde Resident for DCH, ${ }^{4}$ Rashmi \\ Dwivedi MD Professor and Head, Department of Paediatrics. All from Gandhi Medical College and Associated Kamla Nehru \\ Hospital, Bhopal (M.P), India 462016
}

Address for correspondence: Dr Jayashree Nadkarni, Email: jayadn2007@gmail.com

\begin{abstract}
Introduction- Mid-arm circumference (MAC) has been widely used as an age-independent indicator of undernutrition at 1-5 years of age. Its role below one year of age has not been extensively studied. Growth faltering which predicts protein-energy malnutrition (PEM) usually begins between ages 6 and 12 month. This study aimed to determine the age-independent character of MAC within 7-11 month age group and search for a reliable cut off point. Methods- This institutional based, cross-sectional, observational study was done at three government hospitals including a teaching tertiary level hospital. Study variables were age, sex, height, body weight \& mid arm circumference. Mid arm circumference was validated against weight for age percentage and Standard deviation score. Results- Mean MAC of the infants increased very little with age from 7-11months. The cut off points from 13.0 to $12.5 \mathrm{~cm}$ showed maximum diagnostic accuracy. Conclusions- MAC measurement is a simple and valid tool for detection of early PEM in this crucial age bracket However, further studies are suggested, preferably community based and in a larger sample to establish the validity of MAC to detect PEM in infants.
\end{abstract}

Key words: MAC, PEM, infants

\section{Introduction}

Malnutrition in infancy often goes undetected in developing countries due to deficiency of healthcare facilities and lack of simple and feasible screening techniques. Detection and early intervention at this stage can correct the nutritional deficiencies. Peripheral healthcare workers need a simple and feasible technique to detect malnutrition particularly when a functioning weight machine is not always available. The MAC is a

Manuscript received: $13^{\text {th }}$ Sep 2013

Reviewed: $26^{\text {th }}$ Sep 2013

Author Corrected: $29^{\text {th }}$ Sep 2013

Accepted for Publication: $30^{\text {th }}$ Sep 2013 simple, sensitive and cost effective tool for community assessment of nutritional status in children above six months of age ${ }^{1,2,3,4}$. Its measurement is relatively easy to perform and requires minimal time and effort.

Growth faltering, which may indicate protein-energy malnutrition (PEM) usually begins between ages 6 and 12 months ${ }^{1,2}$.

In breast fed babies malnutrition may start around six months of age as improper complimentary feeds may be introduced and there may be sudden withdrawal of breast feeds ${ }^{5}$. Intercurrent infections like diarrhoea and acute respiratory infections also hasten 
malnutrition. Most of the PEM are mild to moderate and may go unnoticed unless it is specifically looked for. Thus, identification of malnutrition as early as possible in this period is crucial.

A recent WHO statement recommends MUAC cut offs of $<115 \mathrm{~mm}$ in children aged 6-60 months for identifying severe acute malnutrition.

Weight for height $<-3$ SD, MUAC $<115 \mathrm{~mm} \&$ bilateral oedema have been considered as independent indicators of SAM that require urgent action ${ }^{6}$.

Mid-arm circumference (MAC) has thus been extensively used at 1-5 years of age as an age-independent indicator of protein-energy malnutrition.

Studies to detect malnutrition by measuring Mid Arm Circumference during second half of infancy are very few $1,2,3,5,7,8$

A search for a clinically reliable cut-off point has not been systematically done. In fact, MAC may be the single available tool, which can help health workers to identify malnutrition at an early stage.

Mean MAC does not rise substantially during the second half of infancy as proposed by researchers and so can be deemed to be an age independent variable from 6 months onwards.

A single cut off value, if found, can be useful for early detection of malnutrition. The present study was done to assess if MAC is a useful tool for early detection of malnutrition among 7-11 month old infants and identify its validity characteristics in this age group.

\section{Material \& Method}

We conducted an urban hospital based, observational, cross sectional study.178 infants randomly selected from OPD \& immunization clinics of Kamla Nehru Hospital, Jai Prakash Hospital and Government Hospital, Bairagarh were the study group.
Study was carried on between June 2011 to December 2011. Only babies who were brought for routine monthly check up and immunization were included.

Each infant selected for the study was weighed on a digital weighing machine noted to nearest of $100 \mathrm{gm}$.

(Weight was taken by a Salter's balance, Model 235PBW) and the calibration of the weighing scale was checked daily with a known weight.

The MAC measurements were taken by a flexible nonstretchable tailor's cloth tape, on the left upper arm at the point midway between the tip of the acromian process of scapula and tip of olecranon process of ulna, and were recorded to nearest $1 \mathrm{~mm}^{8}$.

All variables like age, sex, residence and examination findings were noted in a pre-designed Performa.

Infants between 7-11months with/without malnutrition were arranged according to MUAC values. The values at and above the cut off points were considered as normal \& the values below were considered abnormal.

The position of the cut-off points determined the Total Positive, Total Negative, Positive Predictive Value, Negative Predictive Value \& diagnostic accuracy $(2 \times 2$ contingency table).

Data was collected, tabulated \& analysed on SPSS16 (Statistical Package for Social Sciences 16). In this study "PEM" was taken as body weight less than mean-2SD for that particular age ${ }^{9}$.

\section{Results}

178 infant in age group of 7-11 months attending OPD and immunization clinics of above mentioned hospitals from June 2011 to Dec 2011 were studied.

Out of 178 infants, 88 (49.44\%) were male (Table No.1). Prevalence of PEM (i.e. weight < "mean-2SD limits" for the age) was $43(24.16 \%)$. 
Table No.1: Age and Sex wise distribution of cases

\begin{tabular}{|c|c|c|c|c|c|c|c|c|c|c|c|}
\hline $\begin{array}{c}\text { Age } \\
\text { (months) }\end{array}$ & \multicolumn{3}{|c|}{ Male } & \multicolumn{3}{c|}{ Female } & \multicolumn{3}{c|}{ Total } & $\begin{array}{c}\text { Sex } \\
\text { difference } \\
\text { (t value) }\end{array}$ & $\begin{array}{c}\text { P } \\
\text { value }\end{array}$ \\
\hline & No & Mean & SD & No. & Mean & SD & No. & Mean & SD & \\
\hline 7 & 15 & 13.09 & 0.59 & 17 & 12.71 & 0.56 & 32 & 12.80 & 0.64 & 1.87 & 0.07 \\
\hline 8 & 17 & 13.05 & 0.67 & 18 & 13.01 & 0.51 & 35 & 13.03 & 0.62 & 0.25 & 0.80 \\
\hline 9 & 14 & 13.09 & 0.67 & 17 & 13.12 & 0.44 & 31 & 13.11 & 0.58 & 0.10 & 0.10 \\
\hline 10 & 20 & 13.24 & 0.64 & 21 & 13.17 & 0.38 & 41 & 13.20 & 0.54 & 0.37 & 0.37 \\
\hline 11 & 22 & 13.35 & 0.59 & 17 & 13.33 & 0.34 & 39 & 13.34 & 0.51 & 0.19 & 0.85 \\
\hline Total & 88 & 13.18 & 0.59 & 90 & 13.07 & 0.56 & 178 & 13.13 & 0.64 & 1.28 & 0.20 \\
\hline
\end{tabular}

Mean MAC of the infants increased very little with age from 7-11months. The overall increase in MUAC was $0.44 \%$ of total, $(0.26 \%$ for males $\& 0.62 \%$ for females $)$ and these changes were found to be statistically insignificant $(p>0.05)$.

Male infants between 7-11months in general possessed higher mean value for MUAC than their female counterparts except at 10 months of age. Magnitude of sex difference in 7-11 months was statistically insignificant ( $\mathrm{p}<=0.05)$. We did not find any differences between the measurements taken at the three hospitals.

Table No.2 \& Figure I shows the validity characteristics of different levels of MAC to detect PEM among the age group 7-11 month infants.

The cut off points from 13.0 to $12.5 \mathrm{~cm}$ showed maximum diagnostic accuracy of about $86 \%$ suggesting a correct diagnosis (i.e. true positive \& true negative) of PEM. At $13 \mathrm{~cm}$ cut off point, a high sensitivity of $67.44 \%$ and specificity of $91.85 \%$ was noted, while on the other hand at $12.5 \mathrm{~cm}$, sensitivity of about $51.16 \%$ (true positive) and the specificity was $96.30 \%$ ( leaving very few false positive/ misdiagnosis) and with overall correct diagnosis of around $85.39 \%$.

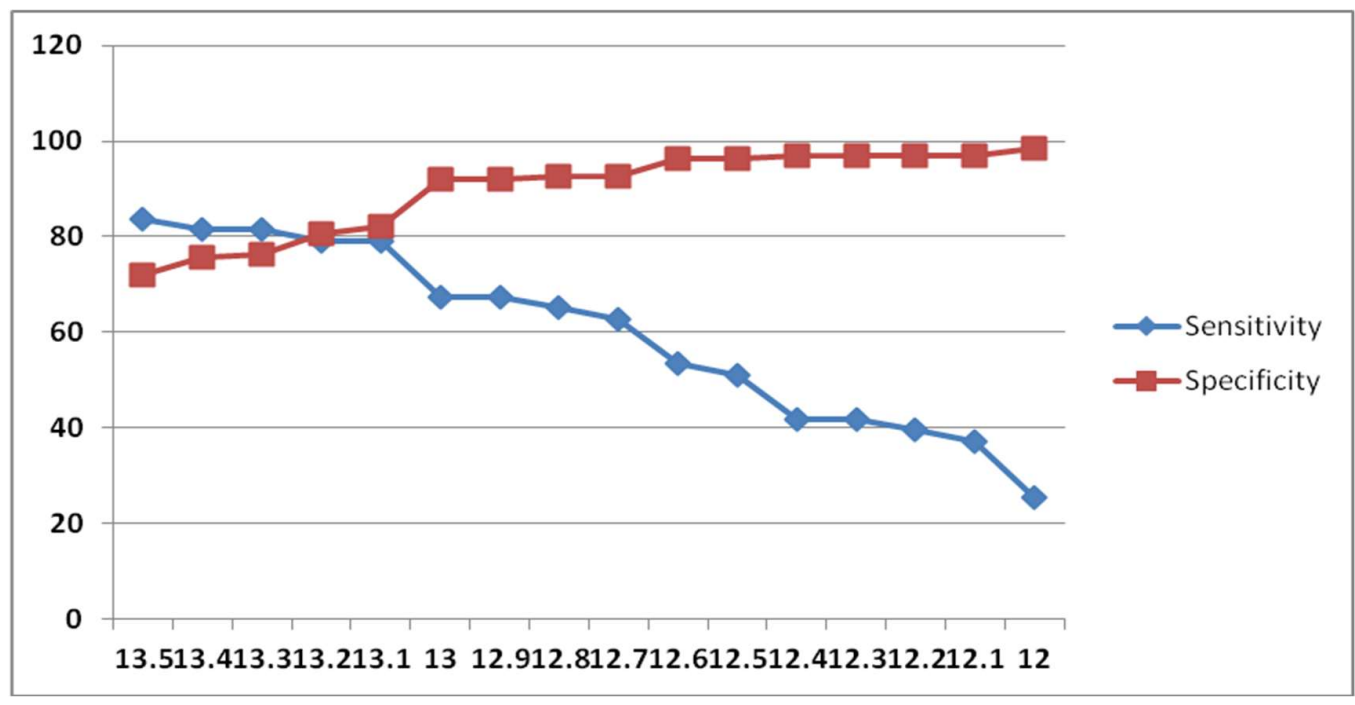


Table No.2: Validity characteristics of different levels of MAC to detect PEM among 7-11 month Infants

\begin{tabular}{|c|c|c|c|c|c|}
\hline \multicolumn{6}{|c|}{ Validity characteristics of different levels of MAC to detect PEM (n=178) } \\
\hline $\begin{array}{l}\text { MAC cut } \\
\text { off(cms) }\end{array}$ & Sensitivity & Specificity & $\begin{array}{c}\text { Positive Predictive } \\
\text { value }\end{array}$ & $\begin{array}{c}\text { Negative } \\
\text { Predictive value }\end{array}$ & $\begin{array}{r}\text { Diagnostic } \\
\text { accuracy }\end{array}$ \\
\hline 13.5 & 83.72 & 71.85 & 48.65 & 93.27 & 74.72 \\
\hline 13.4 & 81.40 & 75.56 & 51.47 & 92.73 & 76.97 \\
\hline 13.3 & 81.40 & 76.30 & 52.24 & 92.79 & 77.53 \\
\hline 13.2 & 79.07 & 80.74 & 56.67 & 92.37 & 80.34 \\
\hline 13.1 & 79.07 & 82.22 & 58.62 & 92.50 & 81.46 \\
\hline 13 & 67.44 & 91.85 & 72.50 & 89.86 & 85.96 \\
\hline 12.9 & 67.44 & 91.85 & 72.50 & 89.86 & 85.96 \\
\hline 12.8 & 65.17 & 92.59 & 73.68 & 89.29 & 85.96 \\
\hline 12.7 & 62.80 & 92.59 & 72.97 & 88.65 & 85.39 \\
\hline 12.6 & 53.49 & 96.30 & 82.14 & 86.67 & 85.96 \\
\hline 12.5 & 51.16 & 96.30 & 81.48 & 86.09 & 85.39 \\
\hline 12.4 & 41.86 & 97.04 & 81.82 & 83.97 & 83.71 \\
\hline 12.3 & 41.86 & 97.04 & 81.82 & 83.97 & 83.71 \\
\hline 12.2 & 39.53 & 97.04 & 80.95 & 83.44 & 83.15 \\
\hline 12.1 & 37.21 & 97.04 & 80.00 & 82.91 & 82.58 \\
\hline 12 & 25.58 & 98.52 & 84.62 & 80.61 & 80.90 \\
\hline
\end{tabular}

\section{Discussion}

The present study shows that MAC of an infant within 711 months shows very little change. We observed an overall increase in MAC of $0.44 \%$. Similar findings were observed in the study conducted by Bhalla $\mathrm{AK}^{2}$ amongst Punjabi infants aged 8-11 month of age, by Martorell $\mathrm{R}$ et $\mathrm{al}^{10}$ among Guatemalan infants aged 9-12months \& by Sadhukhan SK et $\mathrm{al}^{5}$ among Bengali rural infants.

Thus, the study supports the finding that mean MAC of infants was almost constant and could be considered to be almost age independent.
It was observed that MAC level from 13 to $12.5 \mathrm{~cm}$ had the maximum diagnostic power (about 86\%) and MAC level of $12.5(12.6) \mathrm{cm}$ had the minimum false positivity (only around $4 \%$ ).

Also at MAC value of $12.5 \mathrm{~cm}$, the positive \& negative predictive values of $81.5 \%$ \& $86.1 \%$ were favourable with a very high likelihood ratio of 13.9 (close to maximum value of 17.3). In one study it was observed that MAC level from 13 to $12.5 \mathrm{~cm}$ had the maximum diagnostic accuracy ${ }^{5}$. 
MAC of $12.5 \mathrm{~cm}$ produced a high sensitivity \& specificity in another study too ${ }^{2}$. Bhatia $\mathrm{RC}$ et $\mathrm{al}^{3}$ found MAC to be age independent with overall increase of $5.1 \%$. A value of $12.5 \mathrm{~cm}$ had the maximum specificity in their study.

Pust $\mathrm{R}$ et $\mathrm{al}{ }^{1}$ observed that $\mathrm{MAC}<12.5 \mathrm{~cm}$ may provide a simple \& valid screening test for early PEM between 6-12 months of age. One study found that MAC, with the addition of bipedal oedema, was found to be the indicator best suited for screening $\&$ case detection of malnutrition in the community ${ }^{4}$.

In our study, with the use of MAC alone at $12.5 \mathrm{~cm}$, around $51 \%$ of undernourished infants could be detected. Thus, MAC measurement can have many practical advantages over the standard anthropometry, especially at the community level as has been documented in other studies $^{1,3}$.

\section{Conclusions}

MAC is, therefore, a simple, age independent, cost effective and measurement for early assessment of PEM by using single cut-off point and can serve as a screening test for early detection of malnutrition in infants.

MAC can provide a viable alternative to other cumbersome methods in use in field surveys.

Early intervention in such children can prevent their progression to more severe grades of malnutrition. It can thus be put to use in community for meaningful utilization of nutritional programmes.

However, we suggest further studies, preferably community based and in a larger sample to establish the validity and threshold of MAC to detect malnutrition.

\section{Acknowledgement}

We acknowledge support of Medical Superintendents of Jai Prakash Hospital and Bairagarh Government Hospital and Dr Smita Saxena, Specialist Paediatrics, Bairagarh Hospital for their valuable help.

\section{Funding: Nil}

\section{Conflict of interest: Nil}

\section{Permission from IRB: Yes}

\section{References}

1. Pust R, Johnson P, Lautenschlager J. Detecting malnutrition at age 6-12 months: international comparisons of arm circumference v. standard anthropometry. J Trop Pediatr. 1992 Oct; 38(5):240-6.

2. Bhalla AK. Longitudinal Growth of Arm Circumference in Punjabi Infants. Indian Pediatr. 1999 Mar; 36(3):257-62.

3. Bhatia RC, Pooni PA, Singh H, Singh D. Mid-arm circumference for detecting malnutrition during infancy. Indian J Pediatr.1999 Nov-Dec; 66(6):843-8.

4. Myatt M, Khara T, Collins S. A review of methods to detect cases of severely malnourished children in the community for their admission into community-based therapeutic care programs. Food Nutr Bull. 2006 Sep; 27(3 Suppl):S7-23.

5. Sadhukhan SK, Chatterjee C, Shrivastava P, Sardar JC, Joardar GK, Lahiri S. Validity of mid arm circumference to detect protein energy malnutrition among 8-11 months old infants in a rural medical college of West Bengal. J Indian Med Assoc. 2010 Sep; 108(9):559-62.

6. Physical status: the use and interpretation of anthropometry. Report of a WHO Expert Committee. World Health Organ Tech Rep Ser. 1995;854:1-452

7. Jelliffe DB, Jelliffe EF. Age-independent anthropometry. Am J Clin Nutr.1971 Dec;24(12):1377-9.

8. Yost DA, Pust RE. Arm circumference as an index of protein - energy malnutrition in 6-11 month old rural Tanzanian children. J Trop Pediatr. 1988 Dec;34(6):27581. 
9.www.WHO.int/childgrowth.standards/weight_for_age/e $\mathrm{n} /$ index.html
10. Martorell R, Yarbrough C, Lechtig A, Delgado H, Klein RE. Upper arm anthropometric indicators of nutritional status. Am J Clin Nutr. 1976 Jan;29(1):46-53.

\section{How to cite this article?}

Nadkarni J, Sharma U, Bagde R, Dwivedi R. Detection of Malnutrition in Older Infants by Measurement of Mid arm Circumference. Int J Med Res Rev 2013;1(4):156-161. doi:10.17511/ijmrr.2013.i04.03 